\title{
Proactive interference, recency, and associative strength: Comparisons of black-capped chickadees and dark-eyed juncos
}

\author{
ROBERT R. HAMPTON, SARA J. SHETTLEWORTH, and RICHARD P. WESTWOOD \\ University of Toronto, Toronto, Ontario, Canada
}

\begin{abstract}
Black-capped chickadees (Parus atricapillus) and dark-eyed juncos (Junco hyemalis) were required to match to the last item from a series of up to three stimuli differing in both location and color. When rewarded for pecking the target stimulus during the study phase of each series, black-capped chickadees demonstrated proactive interference (PI) from stimuli presented prior to the target, whereas juncos did not. When they made an error, chickadees were more likely than were juncos to choose a distractor from the study series rather than a novel stimulus. When reward was no longer associated with presentation of the final target sample in a series, juncos also suffered PI. These results indicate that chickadees and juncos differ in the degree to which the recency of stimuli and the associative strength of stimuli control correct matching.
\end{abstract}

Over the past 15 years, scatter-hoarding birds have been the focus of numerous studies aimed at characterizing specializations of memory that are thought to be related to their dependence on memory for many individual food cache sites (Clayton, 1995; Krebs, 1990; Sherry, Jacobs, \& Gaulin, 1992; Shettleworth, 1995). Enlargement of the hippocampus in food-storing birds is a well-documented specialization of the brain that presumably underlies enhancement of some aspect of memory related to the retrieval of cached food (see Clayton \& Krebs, 1995; Krebs, Sherry, Healy, Perry, \& Vaccarino, 1989; Sherry, Vaccarino, Buckenham, \& Herz, 1989). However, in contrast to the reliability with which hippocampus volume is related to scatter hoarding, efforts to document superior memory in scatter-hoarding birds have met with mixed results (see Shettleworth, 1995). It may be that research on quantitative differences between species has been emphasized, whereas possible qualitative differences in how species solve memory tasks have been overlooked.

Some research has revealed apparently simple quantitative relationships between food storing and the accuracy and duration of memory (see, e.g., Kamil, Balda, \& Olson, 1994; Olson, 1991). Other work has revealed situations in which storing and nonstoring species achieve equivalent levels of accuracy but do so by different means (see, e.g., Brodbeck, 1994; Clayton \& Krebs, 1994a, 1994b). Foodstoring birds preferentially respond to the absolute location

This work was supported by a research grant from the Natural Sciences and Engineering Research Council of Canada to the second author. We thank Andrew Gristock and Mike Child for conscientious and dedicated animal care. Tim Jarsky and Joel Grossman helped test the animals. Correspondence concerning this article should be addressed to R. R. Hampton, who is now at the Laboratory of Neuropsychology, National Institute of Mental Health, Building 49, Room IB-80, Bethesda, MD 20892 (e-mail: robert@ln.nimh.nih.gov). of a feeder containing a reward, whereas nonstoring birds do not respond more to location than to other properties, such as color (see Healy, 1995, for a possible exception). These results highlight the importance of comparing how each species solves a given task, in addition to how well they perform.

One quantitative difference that has received some support is that memory in food-storing birds may be especially resistant to proactive interference (PI; Clayton \& Krebs, 1994b; Hampton \& Shettleworth, 1996b). PI is inferred whenever information presented to subjects prior to the information that they must retain in memory interferes with their ability to retain the target information (Wright, Urcuioli, \& Sands, 1986). Clayton and Krebs (1994b) required birds to find hidden food and tested the animals' memory for the location of the food after a retention interval. Two food-storing species were generally able to return directly to the site at which food had been found previously, whereas two nonstoring species searched with equal probability in the correct site and in sites that they had visited while initially searching for the food. The nonstoring species appear to be more affected by PI from incorrect sites visited while searching for the target (Clayton \& Krebs, 1994b).

In another demonstration of species differences in susceptibility to PI, black-capped chickadees and darkeyed juncos (a species that does not store food) were tested in operant two-choice spatial nonmatching-tosample (Hampton \& Shettleworth, 1996b). Juncos tended to respond to the pecking key rewarded on the previous trial in this task, whether or not it was the correct response for the current trial, whereas chickadees did not show PI from previous trials. Furthermore, lesions of the hippocampus increased the tendency to choose the previously rewarded key in both species, indicating that this PI effect is related to hippocampal function. Resistance to 
PI has previously been linked to hippocampal function in mammals (Mayes, Pickering, \& Fairbairn, 1987; Shapiro \& Olton, 1994; Winocur, 1979).

To test the generality of the results of Clayton and Krebs (1994b), we designed an operant paradigm similar to their aviary experiment and used North American rather than European storing and nonstoring birds. Testing additional species allows assessment of the reliability of the relationship between the behavior of food storing and resistance to PI. As well as offering the many advantages of automated testing, use of an operant setting tests the generality of proposed species differences in the face of changing contextual variables. If food-storing birds have specialized memory mechanisms that make them generally resistant to PI, food-storing birds should perform better than nonstoring birds in a variety of situations in which resistance to PI is important. Previous work has shown that at least some differences between species obtain in both operant and other environments (reviewed in Shettleworth, 1995).

We simulated searching for food in an array of feeders and manipulated PI, by requiring birds to peck at varying numbers of distractors before presentation of a tobe-remembered sample. We tested memory by presenting the birds with the items they had encountered and, sometimes, additional stimuli that they did not see while "searching" for the sample and requiring them to matchto-sample. If food-storing birds are more resistant to PI from items encountered while searching for a target, as was suggested by Clayton and Krebs (1994b), we should reproduce the finding of impaired performance by nonstoring birds in the face of PI.

We also investigated the relative roles of recency and associative strength in controlling birds' delayed matching performance. Recency refers to the time since a stimulus was last viewed. If subjects view a series of stimuli, the last one viewed would be the most recent, the second to last one viewed would be the next most recent, and so on. Associative strength refers to the degree to which a particular stimulus is associated with reward. Thus, if pecking at or approaching a given stimulus results in a reward, associative strength for that stimulus increases. If pecking a stimulus results in nothing, associative strength for that stimulus decreases. Previous studies with monkeys (Gaffan, 1974) and pigeons (see, e.g., Macphail, Good, \& Honey, 1995) have established that recency and associative strength may both influence performance in delayed matching tasks.

Many tests of memory may be completed successfully by either matching or nonmatching (depending on which rule applies) to the most recently seen stimulus, the sample from the study phase of a trial. In studies comparing memory between storing and nonstoring species (see, e.g., Hampton \& Shettleworth, 1996b; Olson, Kamil, Balda, \& Nims, 1995), animals were not rewarded during study of the sample stimulus. However, in the Clayton and Krebs (1994b) experiment, reward was associated with the tar- get when the birds located hidden food during study and were allowed to eat some of it before the beginning of the retention interval.

Pairing of reward with the sample during study means that the choice of the correct sample at test can result from selecting either the stimulus with the highest associative strength or the stimulus that was seen most recently. In studies of matching- and nonmatching-to-sample in which reward is not paired with any stimulus during study, animals must respond entirely according to which stimulus is remembered as being seen most recently. We contrast these two mechanisms for successful matching between chickadees and juncos across two experiments.

\section{EXPERIMENT 1}

In this experiment, juncos and chickadees were compared in a matching task in which reward was paired with presentation of the target sample during study. PI was manipulated by exposing birds to varying numbers of irrelevant stimuli prior to presentation of a to-be-remembered sample. By the end of training, birds were exposed to series of one, two, or three items and, at test, were rewarded for choosing the last item in the series. On the basis of the results of Clayton and Krebs (1994b), one would predict that chickadees and juncos should do equally well when presented with only one item. These trials are similar to the situation in which the food is visible when birds enter the aviary during the study phase and in which the birds travel directly to the food. However, when presented with series of two or three items, as in the situation in which a bird visits several empty sites before finding the baited one, chickadees should continue to perform well, whereas the performance of juncos should drop off, because of the deleterious effect of PI.

In previous comparisons of chickadees and juncos in similar tasks (see, e.g., Brodbeck \& Shettleworth, 1995; Hampton \& Shettleworth 1996a, 1996b), it has not been possible to compare the species on acquisition of the task, because parameters such as duration of stimulus exposure and retention interval were largely determined by the subjects and differed between the species. When testing spatial memory, it is necessary to require animals to move away from the computer monitor during the retention interval, so that they cannot stand in front of the correct location waiting for the test stimuli to appear rather than using memory. We required birds to break a photobeam in the back of the test box to terminate the retention interval and initiate tests. When the retention interval is programmed to be $0 \mathrm{sec}$ and animals can respond at their own pace, juncos typically take substantially longer retention intervals than do chickadees (see, e.g., Hampton \& Shettleworth, 1996b). We attempted to correct for this by using a retention interval longer than $0 \mathrm{sec}$ from the $1 \mathrm{st}$ day of training, thus forcing chickadees to take retention intervals more similar to those experienced by juncos. We also tried to equate stimulus exposure by placing minimum 
limits on the number of pecks and duration of exposure in conjunctive fixed ratio, fixed interval (FR-FI) presentation schedules.

\section{Method}

Subjects. Seven black-capped chickadees and 6 dark-eyed juncos were caught at the Erindale campus of the University of Toronto. One bird had stored food in an experiment on seasonality that did not involve tests of memory; the rest of the birds were experimentally naive. The animals lived in individual wire mesh cages $(36 \times$ $36 \times 61 \mathrm{~cm})$ in the room in which the experiment took place and were kept on a 10.5:13.5-h light:dark cycle (lights on at 7:00 a.m.) at $66^{\circ} \mathrm{F}$. When they were to be tested the next day, all food was removed from cages shortly before lights out. Food was withheld until the bird was tested the following day (chickadees between 9:00 and 11:30 a.m., juncos between 10:15 a.m. and 1:30 p.m.).

A maintenance diet, consisting of millet and niger seeds (for the juncos), insectivorous bird food mixed with grated carrot and hard-boiled egg, peanuts, and mealworms, was returned by 1:00 p.m. (chickadees) and 3:00 p.m. (juncos). The additional food types offered juncos are necessary to their health, and the differences in duration of deprivation reflect the fact that juncos are substantially larger than are chickadees. This schedule of deprivation does not necessarily produce "equivalent" levels of hunger but is the result of a balance between the need to motivate each species to perform reliably in the experiments and the need to ensure adequate nutrition and is based on years of experience testing these species. Water was continuously available for drinking and bathing. The animals were tested 5 or 6 days a week and had unrestricted access to food at least 1 full day each week.

Apparatus. The subjects were tested in one of three wire mesh chambers. Each chamber $(62 \mathrm{~cm}$ long $\times 37 \mathrm{~cm}$ high) was wider at one end than at the other. At the front end ( $46 \mathrm{~cm}$ wide), an opening ( $30 \mathrm{~cm}$ wide $\times 20 \mathrm{~cm}$ high) afforded the birds access to a 14-in. flat screen VGA monitor. The monitor was equipped with a Carrol Touch Smartframe (P.O. Box 1309, Round Rock, TX) consisting of a grid of photobeams used to determine the location of pecks to the screen. A thin sheet of nonreflecting Plexiglas covered each monitor screen. At the opposite end of each chamber ( $27 \mathrm{~cm}$ wide), a trap door allowed birds access to the testing chamber.

A strip of metal $(6 \mathrm{~cm}$ wide) spanned the width of each chamber at a point $3 \mathrm{~cm}$ from the front of each cage. In the center of each strip was a $1.5-\mathrm{cm}$ hole, through which the bird had access to food. A food hopper, fixed under the metal strip, could be raised to present a mixture of ground sunflower seed and insectivorous bird food. A photocell placed across the hole detected pecks into the food hopper. Another photocell (with emitter and detector $10 \mathrm{~cm}$ apart) was positioned $44 \mathrm{~cm}$ from the front of the cage at a height of $11.5 \mathrm{~cm}$ from the cage floor to detect the presence of the bird on a perch just below the beam. Two other perches were positioned directly in front of the monitor at heights of 9 and $20 \mathrm{~cm}$ from the floor. A fourth perch was located between the back perch and the food hopper, $26 \mathrm{~cm}$ from the front of the cage. Experimental events were monitored and controlled by computers in an adjoining room.

Stimuli. The stimuli were colored circles $2.5 \mathrm{~cm}$ in diameter. Each stimulus could be 1 of 14 colors (blue, green, cyan, red, magenta, brown, light gray, dark gray, light blue, light green, light cyan, light magenta, yellow, and white, as defined in the Turbo Pascal programming language; Borland Inc., 1990) and could occupy the center of 1 of 20 grid locations on the monitor: 5 in the $x$-dimension and 4 in the $y$-dimension; the grids measured $4.56 \times 4.67 \mathrm{~cm}$. The stimuli were presented in the centers of touch-sensitive areas measuring $3.85 \times 3.92 \mathrm{~cm}$. The grid was constructed so that an equal number of touchframe beams spanned each grid. Each location and each color was used independently for both target and distractor stimuli before being used again. The stimuli therefore differed on each trial but were not trial unique.

Pretraining. Autoshaping was used to train the birds to peck at a white stimulus presented on the monitor in the grid located in the center column, bottom row. For birds that did not autoshape, handshaping was used. Once birds were pecking at the white stimulus, colored stimuli and all grid locations were used.

Acquisition: One-item series, two test stimuli. In the study phase of each trial, the birds were required to interrupt the photobeam in the back of the chamber in order to cause a single colored sample to appear on the screen. The sample remained on until the bird pecked it five times (FR5) and for a minimum duration of $4 \mathrm{sec}$ (FI4). The screen then cleared, the food hopper was raised, and the bird was allowed a 0.5 -sec access to food, measured from the time the bird first broke the photobeam over the food hopper. Breaking the rear beam after a retention interval of $5 \mathrm{sec}$ caused the sample and an additional stimulus of a different color and location to appear. A correct response to the target (defined as at least two pecks in at least $1 \mathrm{sec}$, or conjunctive FR2-FI1) was followed by a 2-sec access to food. A single peck to the distractor was followed by a timeout of $7 \mathrm{sec}$, after which only the target was presented. A correct response to the target was rewarded on these correction trials. Sessions consisted of 40 trials or $45 \mathrm{~min}$, whichever came first. The bird moved on to the next stage after 200 trials.

One- and two-item series. Two types of trials, one-item and two-item, were mixed randomly in this stage. One-item trials were the same as before. On two-item trials, the study phase consisted of a sequence of two stimuli, as follows. Interrupting the rear photobeam caused the first item to appear; a single peck extinguished it. As soon as the rear photobeam was broken again, the sample appeared. A conjunctive response contingency of FR5 and FI4 then resulted in a $0.5-\mathrm{sec}$ access to food. After the end of the 5 -sec programmed retention interval, a break of the rear photobeam caused both stimuli to appear. The target was the stimulus last seen, the one that had been rewarded during study. The birds continued with this treatment for a minimum of 600 trials and until they were correct on $80 \%$ or more of the last 100 one-sample trials.

One-, two-, or three-item series. In order to prepare subjects for tests on which series of up to three items would be followed by a test with the target and two distractor items, birds were given 100 trials in which three stimuli appeared at test, rather than just two. A peck to either incorrect stimulus resulted in timeout, after which the two stimuli that had not yet been pecked reappeared. This process was repeated if the bird pecked the wrong stimulus a second time, so that all trials ended with a rewarded response to the correct sample.

This final stage consisted of three types of trials, randomly mixed: one-item, two-item, and three-item trials. One- and twoitem trials were the same as before. Three-item trials were the same as two-item trials, except that an additional item was presented during study. Pecking the last item was still rewarded with access to food. Figure 1 depicts this final stage of multiple-item tests, in which the birds saw one, two, or three stimuli during study and were always presented with three stimuli at test. The birds stayed in this condition for a minimum of 900 trials and until they were correct on $80 \%$ or more of the last 100 one-sample trials. Half the data from 1 chickadee (C52) were lost during this phase of the experiment because of a computer error (trials 318-471; trials 736-1,009). Although this bird did complete these trials, they cannot be included in the analysis.

Data analysis. In this and all subsequent analyses, percent correct scores were arcsine square root transformed prior to analysis. This transformation is generally useful for normalizing proportion correct data (Kirk, 1982). Retention interval is the median latency between the offset of the target sample and the first peck to a test stimulus. Study time is the median time from the onset of the final, correct sample to the offset of this sample following completion of 


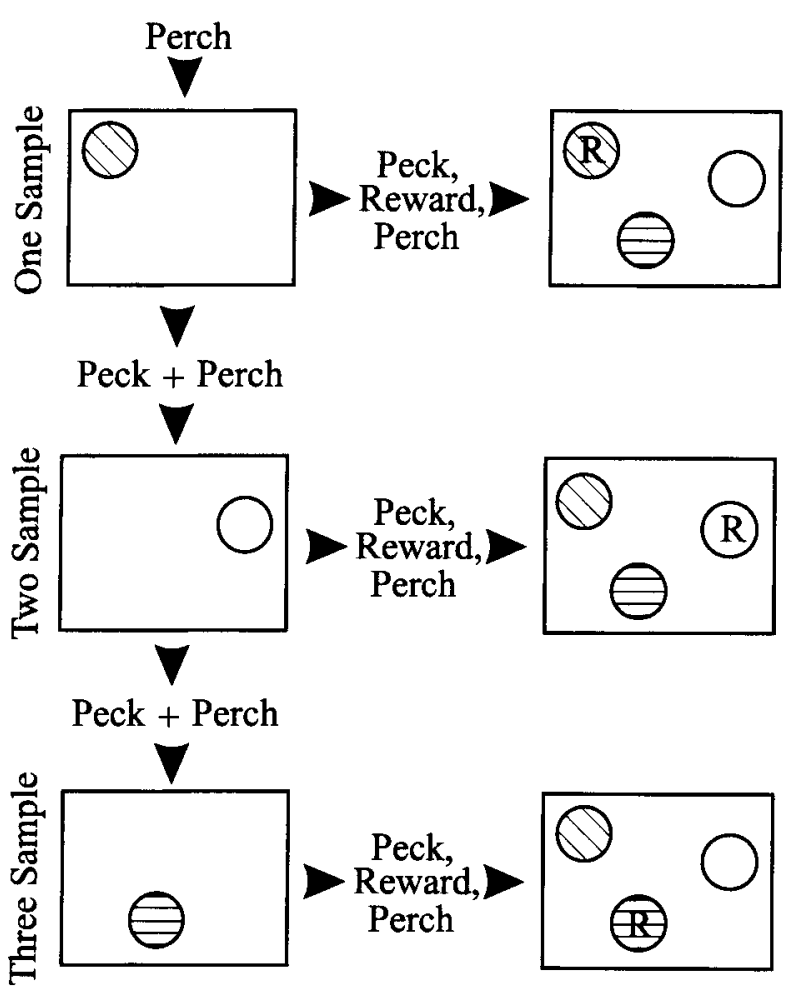

Figure 1. The course of events in trials with one, two, or three samples in the final part of Experiment 1 can be traced by moving down the left-hand column to the appropriate number of samples and then across to the right for the test. $R$ denotes the correct, rewarded, target stimulus. Hatching indicates that the stimuli were different colors. Different colors and locations were used on each trial.

the study response requirement. Effects are considered significant if $p<.05$.

\section{Results and Discussion}

Acquisition. The first 200 trials of training with a single sample and testing with a single distractor were grouped into two blocks of 100 trials each and analyzed using a within-subjects analysis of variance (ANOVA). Both species improved, and they did not differ reliably in accuracy [block, $F(1,10)=9.76, p<.05$; species, $F(1,10)=1.04$; block $\times$ species, $F(1,10)=3.66]$. Despite our efforts at equating retention intervals, juncos experienced substantially longer retention intervals than did the chickadees during the first 200 trials of training, and both species took shorter retention intervals as training progressed. Chickadees experienced retention intervals averaging 22.05 and $15.65 \mathrm{sec}$, respectively, in the first two blocks of 100 trials, whereas juncos experienced retention intervals of 32.88 and $30.47 \mathrm{sec}$ during this time [block, $F(1,10)=5.13, p<.05$; species, $F(1,10)=5.39$, $p<.05$; block $\times$ species, $F(1,10)=1.05]$. Because our efforts at equating retention intervals during acquisition were not successful, we make no further attempts to an- alyze acquisition data and, instead, focus on postcriterion performance, where retention intervals are better matched.

Effect of proactive interference. In the first part of the experiment, chickadees performed somewhat less accurately after exposure to two-item series than after only a single item, whereas juncos did not (Figure 2, left panel). The interaction of species $\times$ number of samples approached significance $[F(1,11)=4.17, p<.10]$. Neither the effect of species $[F(1,11)=0.001]$ nor the effect of number of samples $[F(1,11)=2.75]$ was significant. Juncos experienced slightly longer retention intervals during these tests (Table 1), but the difference was not statistically reliable [species, $F(1,11)=0.34$; number of samples, $F(1,11)=4.79$; species $\times$ number of samples, $F(1,11)=$ $0.62]$. Although chickadees studied the sample slightly longer on average than did juncos (Table 1), analysis indicated no reliable differences in study time [species, $F(1,11)=1.73$; number of samples, $F(1,11)=3.11$; species $\times$ number of samples, $F(1,11)=0.31]$. The trend toward poorer matching accuracy in the chickadees after twoitem series suggests that, if PI were further increased by lengthening the study series, the effect might be stronger.

With series of one, two, or three items intermixed, the performance of chickadees declined substantially as the number of items in the study series increased, but juncos did not show this effect [Figure 2, right panel; species $X$ number of samples, $F(2,18)=7.94, p<.01$; number of samples, $F(2,18)=6.53, p<.01$; species, $F(1,11)=1.00]$. To further substantiate both the PI effect in chickadees and the absence of such an effect in juncos, we compared accuracy on one-sample trials with that on three-sample trials for each species. These paired $t$ tests indicated a reliable difference in chickadees $[t(4)=6.81, p<.01]$ but no difference in juncos $[t(5)=0.35]$. Juncos again took slightly longer retention intervals than did chickadees (Table 1), but the difference was not reliable [species, $F(1,11)=1.11$; number of samples, $F(1,11)=1.48$; species $\times$ number of samples, $F(1,11)=1.23]$. The time each species spent studying the sample decreased slightly, but reliably, as series length increased [Table 1; species, $F(1,11)=0.08$; number of samples, $F(1,11)=5.84, p<$ .05 ; species $\times$ number of samples, $F(1,11)=0.38]$.

Although this experiment differs from that of Clayton and Krebs (1994b) in a number of ways, it captures essential features of their experiment: Birds are exposed to varying numbers of location-color compound stimuli during study, and, at test, they are required to match to the last seen stimulus, which has been paired with reward. Nonetheless, the results are exactly opposite to those from their study. The performance of food-storing chickadees declined when subjects had to "search" for the rewarded sample, whereas in Clayton and Krebs's study it was the nonstoring species that suffered when they had to search. The present results seem to contradict the hypothesis that food-storing birds are more resistant to PI than are nonstoring species (Clayton \& Krebs, 1994b; Hampton \& Shettleworth, 1996b). The close matching 

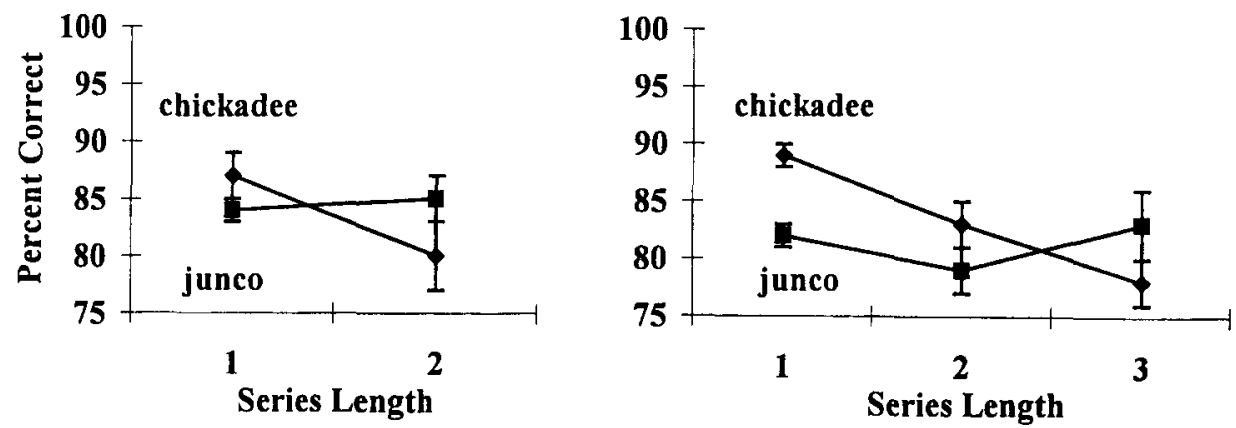

Figure 2. Matching accuracy of chickadees (diamonds) and juncos (squares) in tests with series lengths of up to two stimuli (left panel) or three stimuli (right panel) in Experiment 1. Error bars are standard error.

of birds for retention interval and study time suggests that differences in these parameters are unlikely to account for observed species differences in our study. Although there was a slight but reliable decline in study time with increasing series length, the fact that this occurred in both species and was small in magnitude makes it an unlikely explanation for the PI effect observed in chickadees. It is also unlikely that differences in deprivation could account for differences between our species or for differences between the present experiment and others. What little previous work has been done on the effects of deprivation and reward size in similar tasks has found no effects (Krebs, Healy, \& Shettleworth, 1990). Possible reasons for differences between the results of the present study and those published previously are discussed more fully in the General Discussion section.

Analysis of errors. Recency and associative strength make distinct predictions about which errors should be most common on trials in which the birds saw two samples during the study phase of the trial. In the study phase of these trials, the birds first pecked at one stimulus without reward (the familiar stimulus) and then at a second stimulus paired with reward (the target stimulus). At test, the animals are confronted with these two stimuli plus a third, novel, stimulus (the novel stimulus). An animal responding on the basis of recency should be most likely to respond incorrectly to the familiar stimulus, because it is the second most recently seen stimulus. The novel stimulus either has no recency value (it is treated as new) or else is coded as having occurred a relatively long time ago, on a different trial.

In contrast, when behavior is controlled by associative strength, the novel stimulus should be chosen more often on error trials. The familiar stimulus has a negative associative strength, because pecking it was not followed by reward. The correct stimulus has the highest associative strength, because pecking it was followed by reward. The associative strength of the novel stimulus lies between the negative and the positive, because the animal has no recent experience with that stimulus. Figure 3 depicts the course of events on trials with two-item series and illustrates what is meant by the terms familiar, target, and novel (see, also, Macphail et al., 1995).

Few errors were made following two-item series during the final stage of testing (chickadees, $x=15.0$; juncos, $x=20.7$ ). To more accurately estimate the distribution of errors between the familiar and the novel stimuli, the first 900 trials of the one-to-three-sample, three-test portion of the experiment were collapsed. The number of errors available to analyze increased substantially (chickadees, $x=53.4$; juncos, $x=71.8$ ). Chickadees made a larger proportion of their errors to the familiar stimulus

Table 1

Mean Retention Intervals and Target Study Times

(in Seconds, With Standard Errors) From Two-Stimulus and Three-Stimulus Tests in Experiment 1

\begin{tabular}{|c|c|c|c|c|c|c|c|c|c|c|}
\hline \multirow[b]{4}{*}{ Species } & \multicolumn{10}{|c|}{ Series Length } \\
\hline & \multicolumn{4}{|c|}{ Two-Stimulus Tests } & \multicolumn{6}{|c|}{ Three-Stimulus Tests } \\
\hline & \multicolumn{2}{|c|}{1} & \multicolumn{2}{|c|}{2} & \multicolumn{2}{|c|}{1} & \multicolumn{2}{|c|}{2} & \multicolumn{2}{|c|}{3} \\
\hline & $M$ & $S E$ & $M$ & $S E$ & $M$ & $S E$ & $M$ & $S E$ & $M$ & $S E$ \\
\hline \multicolumn{11}{|c|}{ Retention Interval } \\
\hline Chickadee & 12.34 & 1.14 & 12.06 & 1.00 & 10.79 & 0.71 & 11.04 & 0.85 & 10.50 & 0.76 \\
\hline Junco & 13.53 & 1.6 & 12.94 & 1.42 & 11.83 & 0.78 & 11.98 & 0.75 & 11.94 & 0.78 \\
\hline \multicolumn{11}{|c|}{ Target Study Time } \\
\hline Chickadee & 5.71 & 0.86 & 5.34 & 0.66 & 4.65 & 0.29 & 4.49 & 0.19 & 4.45 & 0.19 \\
\hline Junco & 4.48 & 0.13 & 4.39 & 0.08 & 4.78 & 0.32 & 4.51 & 0.17 & 4.48 & 0.15 \\
\hline
\end{tabular}




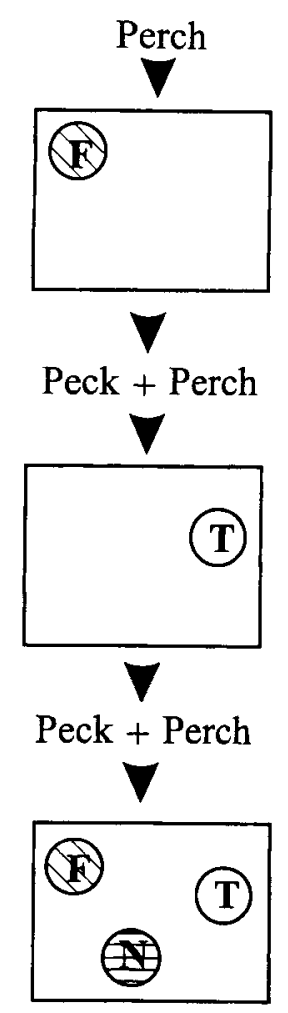

Figure 3. The course of events in a two-item series followed by a three-item test. At the test (bottom panel), the first stimulus that had been presented was familiar (F); the second stimulus presented during study was the target (T). In Experiment 1, but not Experiment 2, reward accompanied the target during study, imbuing it with positive associative strength. The third item in the test display is novel (N).

on two-item trials than did juncos [Figure 4, left panel; $t(9)=2.32, p<.05]$

A second way of testing for a difference in control by recency and associative factors in the two species is to compare accuracy of second choices, after errors to the familiar and the novel stimuli. One source of errors in this task is failure to discriminate between the target stimulus and a distractor because of associative or temporal similarity. Second-choice accuracy should be higher following errors that are due to a failure to discriminate correctly between remembered stimuli (according to either the temporal or the associative strategy) than following errors that are due to forgetting.

An animal using the recency strategy should have higher second-choice accuracy following errors to the familiar stimulus than following errors to the novel stimulus. In contrast, an animal using the associative strategy should perform better on second choices following an error to the novel stimulus. An analysis of secondchoice accuracy following errors to the familiar and novel stimuli on two-item trials supports the hypothesis that chickadees use a temporal strategy and juncos an associative strategy. Chickadees' second choices were most ac- curate following first-choice errors to the familiar stimulus, whereas juncos performed best following errors to the novel stimulus [Figure 4, right panel; species $\times$ first choice, $F(1,9)=6.44, p<.05$; species, $F(1,9)=0.92$; first choice, $F(1,9)=0.46]$.

In summary, chickadees' memory performance declined as the number of items viewed in a series before the target was presented increased. Juncos did not show this effect, indicating that chickadees, but not juncos, were vulnerable to PI from items preceding the target in this operant paradigm. On tests following two-item series, the birds had a choice of the target stimulus, a familiar stimulus, or a novel stimulus. When they made errors, chickadees were more likely than were juncos to choose the familiar stimulus rather than the novel stimulus. In addition, chickadees' second choices were most accurate following errors to the familiar stimulus, whereas juncos were most accurate following errors to the novel stimulus. This pattern of errors and second-choice accuracy indicates that chickadees responded to the stimulus remembered as having been seen most recently, whereas juncos responded to the stimulus most strongly associated with reward.

\section{EXPERIMENT 2 Unrewarded Samples}

In this experiment, we explicitly tested the hypothesis that juncos depend more on associative strength than do chickadees by omitting the reward following the correct sample. In the absence of the associative strength accrued to the sample by reward in Experiment 1, juncos should show decreased accuracy and an increase in PI, because correct responding can be based only on the relative recency of the sample, as compared with the distractors.

\section{Method}

Subjects. Five chickadees and 5 juncos from the previous experiment were maintained as in Experiment 1. Three chickadees and 3 juncos, who experienced a long rest between the end of Experiment $l$ and the start of Experiment 2, were given retraining on oneand two-item series for 50 trials, then one-, two-, or three-item, three-stimulus tests. These retraining trials were conducted exactly as were those in Experiment 1 and included reward following the correct sample. Retraining was complete after at least 300 trials, once the animals had achieved at least $80 \%$ correct on the last 100 onesample trials or when they had completed a maximum of 1,000 trials.

Testing. Testing consisted of trials with one-, two-, or three-item series, followed by three-stimulus tests, exactly as described in Experiment 1 , except that no food was given following responses to the sample during the study phase. This stage continued for 400 trials; 100 of these trials were "warm-up" trials, whereas the last 300 provided 100 trials each of one-, two-, and three-item series.

\section{Results and Discussion}

Retraining. The 3 chickadees that underwent retraining met the criteria in 306,313 , and 389 trials, respectively. In contrast, all 3 juncos completed the maximum of 1,000 retraining trials, and only 1 junco met the crite- 

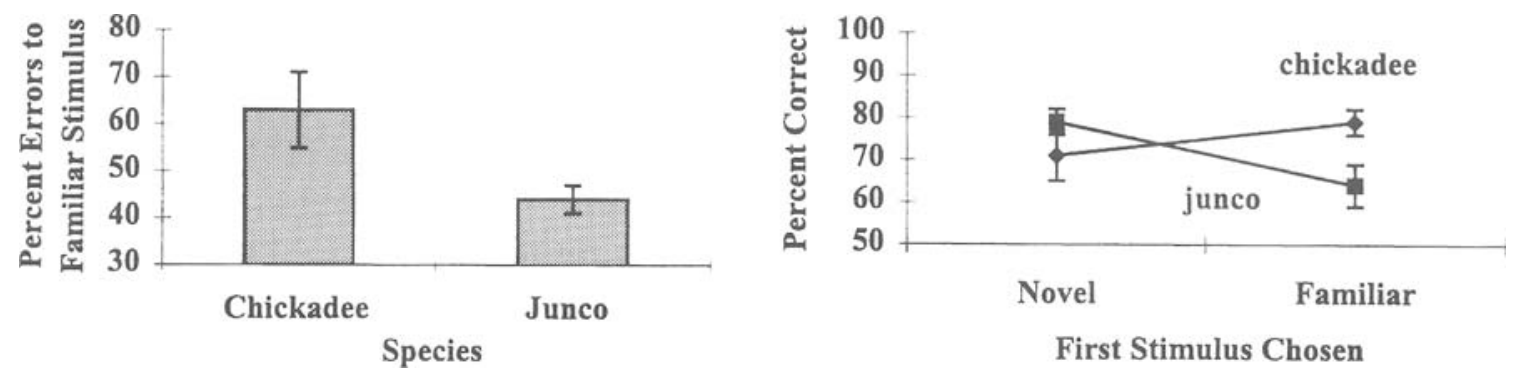

\begin{abstract}
Figure 4. Left panel: Errors were made to one of the two-distractor stimuli on tests with three stimuli, in the second half of Experiment 1. The distractor stimuli presented following sample series of two stimuli are identified as either familiar, the sample that was seen but incorrect on two sample trials, or novel, the sample not seen during study. Right panel: Following errors, the animals were permitted to choose again from the two stimuli not chosen already. Accuracy is depicted as a function of which stimulus was chosen in error during the first choice. The data shown are only from tests using a series length of two stimuli.
\end{abstract}

rion performance level of $80 \%$. An analysis, including only the 3 birds of each species that received retraining, showed that they did less well during retraining than they had done during the last stage of testing in Experiment 1 and that the two species did not differ significantly in the drop in accuracy [phase, $F(1,4)=39.48, p<.01$; species, $F(1,4)=5.21$; phase $\times$ species, $F(1,4)=1.18$ ]. Poor performance during retraining was probably due to the fact that birds took longer retention intervals during retraining than they did during Experiment 1: Retention intervals for chickadees increased by an average of $2.45 \mathrm{sec}$, whereas retention intervals for juncos increased by $8.6 \mathrm{sec}$.

During testing with unrewarded samples, retention intervals taken by both chickadees and juncos were similar to those found in Experiment 1. A comparison of retention intervals taken during the last 300 trials of retraining with those from the last 300 trials of testing with unrewarded samples indicates that retention intervals of both species decreased between these two phases of testing [phase, $F(1,8)=15.82, p<.01$; species, $F(1,8)=2.49$; phase $\times$ species, $F(1,8)=1.06]$. In fact, a comparison of retention intervals from Experiment 1 with those from testing with unrewarded samples showed that both species took shorter retention intervals during the final phase of testing with unrewarded samples [phase, $F(1,8)=51.40$, $p<.01$; species, $F(1,8)=1.81$; phase $\times$ species, $F(1,8)=$ $0.03]$. The decrease in retention interval is probably due to the fact that birds were not rewarded for pecking the sample. The absence of reward following the sample in the present experiment could have contributed to shortening the retention interval in three ways: (1) Reward in Experiment 1 could produce a transient drop in motivation following the sample; (2) birds get fewer rewards per session in this experiment and are, thus, less satiated overall; or (3) eating time $(0.5 \mathrm{sec})$ is no longer part of the retention interval. One would expect these shorter retention intervals, taken during this final stage of testing, to lead to better performance than that in Experiment 1 .

Unrewarded samples. When reward was no longer paired with the correct sample, chickadees performed better than juncos, and the species suffered equally from PI [Figure 5; samples, $F(2,16)=22.46, p<.01$; species, $F(1,8)=17.34, p<.01$; samples $\times$ species, $F(2,16)=$ 2.47]. We substantiated the PI effect in both species, as we did in Experiment 1, by comparing performance on trials with series length one and three for each species. These comparisons indicated reliable differences in both species [chickadees, $t(4)=5.21, p<.01$; juncos, $t(4)=4.66$, $p<.01]$. The magnitude of the difference in retention intervals between the species was similar to that seen previously (about $1 \mathrm{sec}$; compare values in Tables 1 and 2). However, the difference was significant here, whereas it was not before [species, $F(1,8)=10.39, p<.05$; samples, $F(2,16)=2.57$; species $\times$ samples, $F(2,16)=0.98]$. On these trials with unrewarded samples, there was a substantial and reliable difference between the species in study time, and both species studied the target for slightly less time the later it appeared in the series [Table 2; species, $F(1,8)=10.22, p<.05$; number of samples, $F(2,16)=$ $4.04, p<.05$; species $\times$ number of samples, $F(2,16)=$ 1.59]. The fact that this increase in study time correlates with the emergence of PI in juncos suggests that it is a possible explanation for the change in PI. However, increases in study time improve matching performance in pigeons (Roberts \& Grant, 1974), so one would expect that increases in study time would decrease PI by improving memory for the target.

Errors. We examined the frequency of errors to the familiar stimulus on trials of series length two, as in Experiment 1 . To maximize the number of error trials in the analysis, we included all 400 trials with unrewarded samples, including the 100 warm-up trials excluded from the previous analyses. In accord with the fact that both species demonstrate PI in the present experiment, both species selected the familiar stimulus in error more often than would be expected to occur by chance [Figure 6 , left panel; chickadees, $t(4)=4.41, p<.05$; juncos, $t(4)=$ $8.26, p<.01]$. The two species did not differ in the frequency with which they erred to the familiar stimulus $[t(8)=1.93]$. This is consistent with the hypothesis that, 


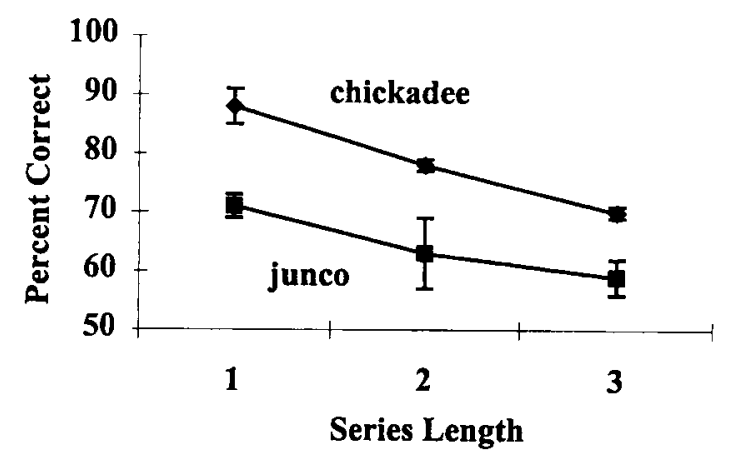

Figure 5. In Experiment 2, reward was no longer provided after responses to the sample during the study phase of the trial. Accuracy is shown for the two species as a function of series length. Decreasing accuracy with increasing series length indicates proactive interference.

in the absence of the positive associative strength accrued to rewarded samples in Experiment 1, both species depend on recency to solve this task. However, there were no significant differences in second-choice accuracy as a function of first choice, in contrast to what was observed in Experiment 1 [Figure 6, right panel; species, $F(1,8)=0.71$; first choice, $F(1,8)=0.01$; species $\times$ first choice, $F(1,8)=$ $0.67]$.

When reward no longer followed responses to the target during the study phase, both species suffered from PI. Examination of the pattern of errors displayed by juncos supports the hypothesis that, between Experiment 1 and Experiment 2, these birds shifted from dependence on a discrimination based on associative strength toward one based on recency. In Experiment 1, only chickadees were more likely to make errors to the familiar stimulus than to the novel stimulus. In Experiment 2, this difference was no longer present, and both species made more errors to the familiar stimulus.

We also found a large species difference in matching accuracy in the present experiment, with chickadees performing better than juncos at each series length. Interpretation of this difference is complicated by the fact that 2 of the juncos that received retraining prior to Experiment 2 never met the criterion of $80 \%$ correct on one-sample trials before moving on to Experiment 2. During retraining, these 2 birds took retention intervals more than two times greater than those they experienced in Experiment 1 . However, by the time of the critical tests with unrewarded samples in Experiment 2, these birds experienced retention intervals shorter than those from Experiment 1. During these critical tests, juncos took retention intervals about $1 \mathrm{sec}$ longer than those taken by chickadees, as in Experiment 1 . In contrast with Experiment 1, in the present experiment, the difference between species in retention interval was statistically significant. However, the magnitude of the difference in retention interval between juncos and chickadees did not change between Experiments 1 and 2. This suggests that the difference between the species in Experiment 2 is due to the lack of the associative information juncos used to solve the task in Experiment 1. It seems unlikely that the small difference in retention interval between the species caused a difference in the use of recency or associative properties of the test stimuli.

\section{GENERAL DISCUSSION}

In Experiment 1, reward was associated with presentation of the target stimulus, and juncos did not show PI. In Experiment 2, reward during the study phase of each trial was omitted, and juncos began to show PI from items preceding the target in the study series. Chickadees showed PI in both experiments, indicating that, even when reward is associated with the target stimulus, recency rather than associative strength controls their choices at test.

When reward was no longer associated with study of the target stimulus, both chickadees and juncos made most errors to the familiar, rather than to the novel, stimulus on two-item series. This pattern of error choices further supports the argument that, in the absence of a reward association with the target stimulus, both species choose the stimulus remembered as most recent at test. Thus, juncos use associative strength more than do chickadees, when it is available (Experiment 1); in the absence of this association (Experiment 2), both species use recency.

In Experiment 1, we modeled the aviary study of Clayton and Krebs (1994b) in an automated testing environment. Despite the similarity in the logic of the experiments, our findings are directly opposite theirs. In Experiment 1 , food-storing chickadees, but not nonstoring juncos, showed evidence of PI. In Experiment 2, both species suffered from PI when successful completion of the task depended on recency memory in the absence of associative information, and there was no difference between the species in the magnitude of the PI effect.

In addition, in Experiment 1, food-storing chickadees performed better than nonstoring juncos when PI was absent (one-item series), but this difference was absent in the high-interference condition (three-item series). These results contradict the hypothesis that food-storing birds are generally more resistant to PI than are nonstor-

Table 2

Mean Retention Intervals and Target Study Times (in Seconds, With Standard Errors) From Three-Stimulus Tests in Which the Correct Sample Was Not Rewarded in Experiment 2

\begin{tabular}{|c|c|c|c|c|c|c|}
\hline \multirow[b]{3}{*}{ Species } & \multicolumn{6}{|c|}{ Series Length } \\
\hline & \multicolumn{2}{|c|}{1} & \multicolumn{2}{|c|}{2} & \multicolumn{2}{|c|}{3} \\
\hline & $M$ & $S E$ & $M$ & $S E$ & $M$ & $S E$ \\
\hline \multicolumn{7}{|c|}{ Retention Interval } \\
\hline Chickadee & 7.00 & 0.26 & 6.92 & 0.25 & 6.95 & 0.22 \\
\hline Junco & 7.93 & 0.10 & 7.8 & 0.15 & 7.71 & 0.12 \\
\hline \multicolumn{7}{|c|}{ Target Study Time } \\
\hline Chickadee & 5.64 & 0.44 & 5.27 & 0.36 & 5.09 & 0.37 \\
\hline Junco & 10.77 & 1.75 & 8.78 & 1.13 & 8.72 & 1.06 \\
\hline
\end{tabular}



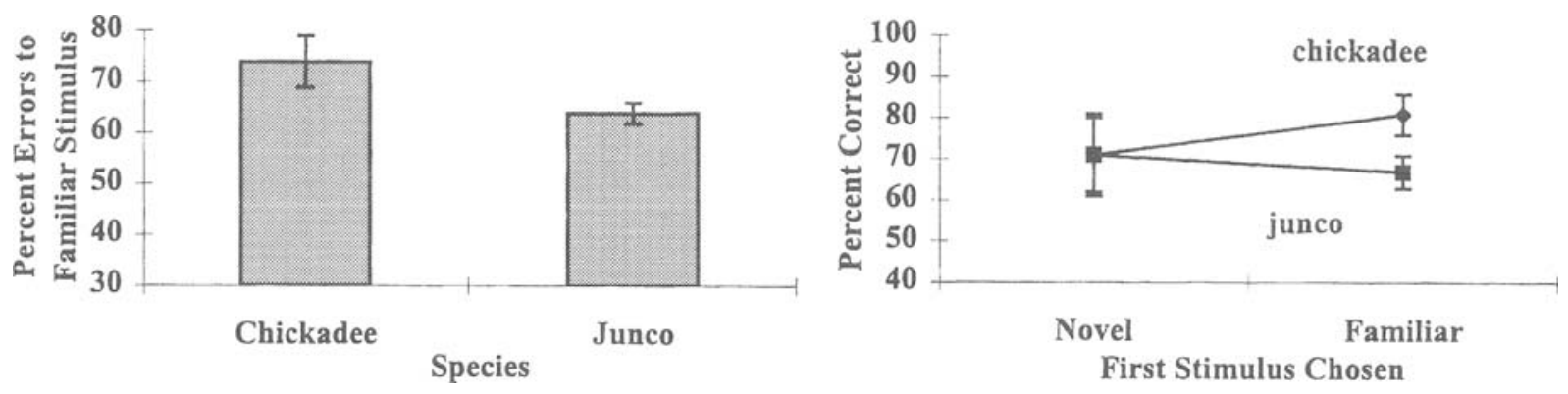

Figure 6. Left panel: The frequency of errors to the familiar stimulus on tests following study series of two stimuli are shown, as in Experiment 1 . One distractor had already been seen and was familiar, whereas the other was novel. Right panel: Second-choice accuracy is depicted as a function of which stimulus was chosen in error during the first choice on tests following a study series of length two.

ing birds. A number of differences between the present experiment and that of Clayton and Krebs (1994b) might account for the apparent conflict in results.

We used different species of storing and nonstoring birds. Although we can specify no specific differences between our species and those of Clayton and Krebs (1994b) that might account for the different results, such differences cannot be ruled out. We controlled the number and order of presentation of distractor items, whereas the birds in the Clayton and Krebs experiment determined the order and number of distractors by their searching behavior. It is, therefore, possible that errors to visited items in their experiment reflected preferences present at both study and test. The birds in the present experiments were tested in the confined space of operant chambers, whereas Clayton and Krebs's birds were tested in the much larger space of an aviary. Test stimuli and other landmarks are further apart in the aviary than they are in operant chambers and may, therefore, be more salient.

In addition, Clayton and Krebs's (1994b) birds experienced much longer retention intervals than did our birds. Increased travel time and richer stimulus environment may result in better performance (see, e.g., Brown, 1990; Crystal \& Shettleworth, 1994), but there seems to be little basis on which to expect qualitatively different results in the two environments. Indeed, both quantitative and qualitative differences between species have been found to hold between testing environments (see Shettleworth, 1995).

Perhaps the most important difference between the experiments is that, in Clayton and Krebs's (1994b) experiment, the birds saw an array of feeders when they entered the room, whereas our birds saw isolated items, one at a time, and were tested with an array composed of the individual items put together. Sequential presentation of stimuli prohibits using the distractor stimuli as landmarks to define the location of the correct sample.

Chickadees and juncos do use distractor stimuli as landmarks in similar tasks, indicated by the fact that birds remember the location of a correct feeder with respect to other feeders in an array of feeders, as well as with respect to more distal landmarks (Brodbeck, 1994). A study in which juncos showed PI but chickadees did not also used a task in which birds saw the sample located in the same spatial array in which tests would occur (Hampton \& Shettleworth, 1996b). The additional information provided by the array of distractor stimuli may improve memory in food-storing birds and protect them from PI.

In a previous study (Hampton \& Shettleworth, 1996b), we concluded that juncos but not chickadees were prone to PI. Although the results of the present experiment indicate that the difference between species in resistance to PI cannot be so broadly described, there is a striking parallel between the present experiment and that of Hampton and Shettleworth. We found that juncos performing spatial nonmatch-to-sample (SNMTS) tended to repeat responses rewarded on the previous trial, whether or not that response was correct on the current trial. Chickadees did not show this pattern of errors. Hippocampus removal caused chickadees to show a strong PI effect, too. One interpretation of this result is that responses are determined by both associative strength and recency memory in SNMTS and that these two sources of response tendency can act in opposition. Furthermore, the fact that hippocampal lesions increase the frequency of reward-based, rather than recency-based, responses indicates that the hippocampus is involved in recency memory and that, when its function is attenuated, associative-based responses are manifested more readily.

In both our experiment and that of Clayton and Krebs, birds were presented with compound stimuli and could, therefore, remember both the location and the color of the stimulus. Food-storing birds base their matching responses largely on the location of a stimulus, whereas nonstoring birds use location and color about equally (Brodbeck, 1994; Brodbeck \& Shettleworth, 1995; Clayton \& Krebs, 1994a). The hippocampus is critical for location but not color memory in juncos and chickadees (Hampton \& Shettleworth, 1996a). The preferential use of space by food-storing birds may underlie differences between jun$\cos$ and chickadees in the use of associative memory.

In rats, hippocampal lesions impair responses based on the location of a platform in a water maze but do not affect performance based on a visible platform (McDonald \& White, 1994). In contrast, lesions of the striatum 
leave animals impaired in attending to the visible platform, but these animals return to the location previously occupied by the platform. It is likely that juncos attended more to the color of our compound stimuli than did chickadees, and this may have, in turn, biased the basis of their responses toward associative, nonhippocampal memory.

Other work indicates that the hippocampus is not required for associative learning, as measured by conditioned place preference in rats (McDonald \& White, 1993; White \& McDonald, 1993) or stimulus preference in monkeys (Gaffan, 1974). In fact, disruptions of the hippocampal system were found to facilitate formation of a conditioned place preference, indicating competition between two memory systems: an associative learning mechanism and the hippocampus (White \& McDonald, 1993). Similar competition between associative and hippocampalbased memory systems may underlie the difference in reaction to interfering stimuli in the present studies.

The fact that juncos and chickadees solve memory tasks differently raises concerns for studies that rely on quantitative differences in performance between species. An assumption of such quantitative comparisons is that species are using the same memory mechanisms. However, if animals use different mechanisms to solve the same task, quantitative comparisons do not relate to the function of a given mechanism. Therefore, it is important to consider qualitative differences between species in an effort to understand the results of comparative studies (see, also, Bitterman, 1975; Brodbeck, 1994; Kamil, 1988).

Much as chickadees use location to find a rewarded stimulus more than do juncos (Brodbeck, 1994; Brodbeck \& Shettleworth, 1995), we suggest that juncos use associative strength more to identify a rewarded stimulus. We do not argue that one type of memory is used to the exclusion of others but rather that these memory systems operate in parallel, sometimes in concert and sometimes in opposition. The combined action of independent memory systems can generate a variety of patterns of differences in comparative studies, with each species showing superior accuracy in different tasks, depending on the extent to which each memory system is engaged (see Kamil et al., 1994, for a related discussion). These patterns of quantitative differences between species are intelligible only when it is known which cognitive mechanisms are brought to bear in each task by each species.

\section{REFERENCES}

Bitterman, M. E. (1975). The comparative analysis of learning. Science, 188, 699-709.

Brodbeck, D. R. (1994). Memory for spatial and local cues: A comparison of a storing and a nonstoring species. Animal Learning \& Behavior, 22, 119-133.

Brodbeck, D. R., \& Shettleworth, S. J. (1995). Matching location and color of a compound stimulus: Comparison of a food-storing and a non-storing bird species. Journal of Experimental Psychology: Animal Behavior Processes, 21, 64-77.

BRown, M. F. (1990). The effects of maze-arm length on performance on the radial-arm maze. Animal Learning \& Behavior, 18, 13-22.
Clayton, N. S. (1995). Comparative studies of food-storing, memory, and the hippocampal formation in parids. Hippocampus, 5, 499-510.

Clayton, N. S., \& KREBS, J. R. (1994a). Memory for spatial and objectspecific cues in food-storing and non-storing species of birds. Journal of Comparative Physiology A, 174, 371-379.

Clayton, N. S., \& KrebS, J. R. (1994b). One-trial associative memory: Comparison of food-storing and nonstoring species of birds. Animal Learning \& Behavior, 22, 366-372.

Clayton, N. S., \& Krebs, J. R. (1995). Memory in food-storing birds: From behavior to brain. Current Opinion in Neurobiology, 5, 149-154.

Crystal, J. D., \& Shettleworth, S. J. (1994). Spatial list learning in black-capped chickadees. Animal Learning \& Behavior, 22, 77-83.

GAFFAN, D. (1974). Recognition impaired and association intact in the memory of monkeys after transection of the fornix. Journal of Comparative \& Physiological Psychology, 86, 1100-1109.

HAMPTON, R. R., \& SHETTLEWORTH, S. J. (1996a). Hippocampal lesion impair memory for location but not color in passerine birds. Behavioral Neuroscience, 110, 831-835.

Hampton, R. R., \& Shettleworth, S. J. (1996b). Hippocampus and memory in a food-storing and in a nonstoring bird species. Behavioral Neuroscience, $110,946-964$.

Healy, S. (1995). Memory for objects and positions: Delayednonmatching-to-sample in storing and non-storing tits. Quarterly Journal of Experimental Psychology, 48B, 179-191.

KAmIL, A. C. (1988). A synthetic approach to the study of animal intelligence. In D. W. Leger (Ed.), Nebraska Symposium on Motivation: Vol. 35. Comparative perspectives in modern psychology (pp. 257308). Lincoln: University of Nebraska Press.

Kamil, A. C., Balda, R. P., \& Olson, D. J. (1994). Performance of four seed caching corvid species in the radial-arm maze analog. Journal of Comparative Psychology, 108, 385-393.

KIRK, R. E. (1982). Experimental design: Procedures for the behavioral sciences (2nd ed.). Belmont, CA: Brooks/Cole.

KREBS, J. R. (1990). Food storing birds: Adaptive specialization in brain and behavior? Philosophical Transactions of the Royal Society of London: Series B, 329, 55-62.

Krebs, J. R., Healy, S. D., \& Shettleworth, S. J. (1990). Spatial memory of paridae: Comparison of a storing and a non-storing species, the coal tit, Parus ater, and the great tit, P. major. Animal Behaviour, 39, 1127-1137.

Krebs, J. R., Sherry, D. F., Healy, S. D., Perry, V. H., \& Vaccarino, A. L. (1989). Hippocampal specialization in food-storing birds. Proceedings of the National Academy of Sciences, 86, 1388-1392.

MaCPhaIL, E. M., GoOD, M., \& HoNeY, R. C. (1995). Recognition memory in pigeons for stimuli presented repeatedly: Perceptual learning or reduced associative interference? Quarterly Journal of Experimental Psychology, 45B, 13-31.

Mayes, A. R., Pickering, A., \& Fairbairn, A. (1987). Amnesic sensitivity to proactive interference: Its relationship to priming and the causes of amnesia. Neuropsychologia, 25, 211-220.

MCDonald, R. J., \& White, N. M. (1993). A triple dissociation of memory systems: Hippocampus, amygdala, and dorsal striatum. $B e-$ havioral Neuroscience, 107, 3-22.

McDonald, R. J., \& White, N. M. (1994). Parallel information processing in the water maze: Evidence for independent memory systems involving dorsal striatum and hippocampus. Behavioral \& Neural Biology, 61, 260-270.

OLSON, D. (1991). Species differences in spatial memory among Clark's nutcrackers, scrub jays, and pigeons. Journal of Experimental Psychology: Animal Behavior Processes, 17, 363-376.

Olson, D., KAMIL, A., BAlDA, R., \& Nims, P. (1995). Performance of four seed-caching corvid species in operant tests of nonspatial and spatial memory. Journal of Comparative Psychology, 109, 173-181.

Roberts, W. A., \& GRant, D. S. (1974). Short-term memory in the pigeon with the presentation time precisely controlled. Learning \& $\mathrm{Mo}$ tivation, 5, 393-408.

ShaPiro, M. L., \& Olton, D. S. (1994). Hippocampal function and interference. In D. L Schacter \& E. Tulving (Eds.), Memory Systems 1994 (pp. 87-117). Cambridge, MA: MIT press.

Sherry, D. F., Vaccarino, A. L., Buckenham, K., \& Herz, R. S. 
(1989). The hippocampal complex of food-storing birds. Brain, Behavior \& Evolution, 34, 308-317.

ShERry, D. F., JACOBS, L. F., \& Gaulin, S. J. C. (1992). Spatial memory and adaptive specialization of the hippocampus. Trends in Neurosciences, 15, 298-303.

ShetTleworTH, S. J. (1995). Comparative studies of memory in food storing birds. In E. Alleva, A. Fasolo, H. Lipp, L. Nadel, \& L. Ricceri (Eds.), Behavioral brain research in natural and semi-natural settings: Possibilities and perspectives (pp. 159-192). Dordrecht: Kluwer.

White, N. M., \& MCDonald, R. J. (1993). Acquisition of a spatial conditioned place preference is impaired by amygdala lesions and improved by fornix lesions. Behavioural Brain Research, 55, 269-281.
Winocur, G. (1979). Effects of interference on discrimination learning and recall by rats with hippocampal lesions. Physiology \& Behavior, 22, 339-345.

Wright, A. A., Urcuroli, R. J., \& Sands, S. F. (1986). Proactive interference in animal memory. In D. F. Kendrick, M. Rilling, \& R. Denny (Eds.), Theories of animal memory (pp. 101-125). Hillsdale, $\mathrm{NJ}$ : Erlbaum.

(Manuscript received December 23, 1997; revision accepted for publication June 26,1998 .) 